\title{
Prognostic significance of combining VEGFA, FLT1 and KDR mRNA expression in lung cancer
}

\author{
SHU-DONG ZHANG ${ }^{1,2^{*}}$, CIAN M. MCCRUDDEN ${ }^{2 *}$ and HANG FAI KWOK ${ }^{1-3}$ \\ ${ }^{1}$ Faculty of Health Sciences (E12), University of Macau, Macau SAR, P.R. China; \\ ${ }^{2}$ Center for Cancer Research and Cell Biology and School of Pharmacy, Queen's University Belfast, Belfast BT9 7BL; \\ ${ }^{3}$ Department of Oncology, University of Cambridge, Cambridge CB2 0XZ, UK
}

Received November 5, 2014; Accepted May 26, 2015

DOI: $10.3892 / \mathrm{ol} .2015 .3415$

\begin{abstract}
Angiogenesis is important in cancer progression. Promising results in clinical trials have indicated that targeting vascular epidermal growth factor (VEGF) signaling may prolong lung cancer patient survival. In particular, various studies have implicated VEGFA as a potential prognostic marker in lung cancer, although prognostication using the expression of VEGF receptors (VEGFRs), such as fms-related tyrosine kinase 1 (FLT1; also known as VEGFR1) and kinase insert domain receptor (KDR; also known as VEGFR2), has produced varied results in different lung cancer studies. The present study aimed to investigate the prognostic significance of these three factors, alone or in combination. mRNA expression data were extracted from four independent lung cancer cohorts totaling 583 patients, and the association between mRNA expression and survival was investigated by performing statistical analyses. When VEGFA, FLT1 and KDR expression were considered alone, only VEGFA demonstrated a significant association with patient survival consistently across all four datasets $(\mathrm{P}<0.05)$. Patients with a high expression of VEGFA and one of the two receptors were associated with significantly worse survival than patients expressing low levels of VEGFA and the particular receptor $(\mathrm{P}<0.05)$. Notably, patients with a high level expression of all three genes in their tumor specimens were associated with a significantly shorter survival time compared with patients exhibiting a low level expression of one, two or all three genes $(\mathrm{P}<0.05)$. The results indicate that a high level of VEGFA expression and its receptors may be required for cancer progression. Therefore, these three factors should be considered together as a prognostic indicator for lung cancer patients.
\end{abstract}

Correspondence to: Professor Hang Fai Kwok, Faculty of Health Sciences (E12), University of Macau, Room 4006, University Avenue, Macau SAR, P.R. China

E-mail: hfkwok@umac.mo; hfk24@cam.ac.uk

*Contributed equally

Key words: lung cancer, vascular epidermal growth factor A, fms-related tyrosine kinase 1, kinase insert domain receptor, angiogenesis, survival

\section{Introduction}

Activation of the vascular epidermal growth factor (VEGF) signaling pathway via interaction between VEGF and VEGF receptors (VEGFRs) has been demonstrated to promote a network of signaling processes that support endothelial cell-mediated angiogenesis (1). Thus, the VEGF pathway is activated via a paracrine action; tumor cells overexpress the ligand, VEGFA, to bind to the receptors, such as fms-related tyrosine kinase 1 (FLT1; also known as VEGFR1) and kinase insert domain receptor (KDR; also known as VEGFR2), which are expressed in the endothelial cells, in order to promote endothelial cell growth and survival, thereby initiating angiogenesis. However, an increasing body of evidence indicates that the VEGF signaling pathway also functions in an autocrine manner, in which tumor cells express VEGF ligand and receptors, and drive intrinsic processes, including tumor cell cycle progression, survival and chemoresistance, ultimately promoting cancer development (2-4).

VEGFA is known to promote angiogenesis through binding to FLT1 and KDR, thus triggering the downstream signaling pathway. Among various pro-angiogenic factors, VEGFA has been identified to be a predominant regulator of tumor angiogenesis (5). High expression of these three genes has been identified in non-small cell lung cancer xenografts compared with xenografts originating from other sites (5), indicating that these three factors may be significant in lung cancer progression. Indeed, VEGFA $(6-8)$, FLT1 $(9,10)$ and KDR $(11,12)$ have all been demonstrated to promote lung cancer progression. However, their utility as prognostic markers remains unknown owing to the conflicting findings of previous studies $(12,13)$.

The aim of the present study was to investigate the prognostic significance of VEGFA, FLT1 and KDR mRNA expression, alone or in combination, in four independent lung cancer patient cohorts from different institutions and of different ethnicities, with a combined patient cohort of 583 patients.

\section{Materials and methods}

Extraction of clinical and microarray gene expression data from lung cancer patient datasets. The GSE3141 (14), GSE4573 (15), GSE8894 (16) and GSE31210 (17) lung cancer patient datasets were identified in the NCBI Gene Expression Omnibus (GEO) 
database (http://www.ncbi.nlm.nih.gov/geo/). Datasets using the HG-U133 microarray platform and comprising of $>100$ patients for whom survival data were available, were included in this study. Microarray gene expression data were retrieved from the data matrixes deposited to the GEO database by the original authors. R scripting was used to extract the expression values from probesets of VEGFA, FLT1 and KDR, and the clinical data was obtained from the data matrixes downloaded from the GEO database, as previously described (18).

Correlation between gene expression levels and clinical data. All statistical analyses were performed using SPSS software (version 19.0; IBM SPSS, Armonk, NY, USA) and P<0.05 was considered to indicate a statistically significant difference. The associations between mRNA expression levels were analyzed by performing Spearman's rank tests. Expression levels were divided into high and low levels using the median expression level as the cut-off point for Kaplan-Meier survival analysis. Results were compared using a log-rank test. Univariate Cox regression analysis was performed to analyze the correlation between mRNA expression levels and patient survival. Median level of gene expression was used as a cut-off to divide patients into high or low expression groups. Patients presenting a target gene expression that was over the median value were classified into the high expression group, while patients with a target gene expression at or below the median value were classified into the low expression group. In addition, patients were divided into 4 groups based on the expression levels of VEGFa, FLT1 and KDR: Patients with a high expression of $0,1,2$ or 3 target genes were classified as 0,1,2 and 3, respectively, for statistical analysis. The survival time of patients stratified by this grouping method were analyzed by Kaplan-Meier and Cox regression analysis, as aforementioned.

\section{Results}

Association between high VEGFA and FLT1 mRNA expression and the survival of patients with lung cancer. As indicated in Table I, Cox regression identified that the VEGFA mRNA expression level was significantly associated with a higher risk of progression or mortality in all four datasets analyzed, with hazard ratios (HRs) ranging between 1.43 and 2.02. Using median VEGFA mRNA expression as the cut-off point, patients with a high level of VEGFA mRNA expression were associated with a shorter survival time. Kaplan-Meier analysis identified a significant association between survival and VEGFA expression in the GSE3141 ( $\mathrm{P}=0.007$; Fig. 1A), GSE4573 ( $\mathrm{P}=0.007$; Fig. 1B) and GSE31210 (P<0.001; Fig. 1D) datasets, but not in the GSE8894 dataset ( $\mathrm{P}=0.182$; Fig. 1C). In the combined datasets $(n=583)$, a high level of VEGFA mRNA expression was identified to be significantly associated with a shorter survival time, according to Kaplan-Meier (log-rank test, $\mathrm{P}<0.001$; Fig. 1e) and Cox regression (HR, 1.94; 95\% CI, 1.50-2.50; P<0.001; Table I) analyses.

For FLT1 mRNA expression, Cox regression analysis identified that high levels of FLT1 mRNA expression were significantly associated with a shorter survival time in two datasets (GSE3141 and GSE8894; P<0.05), but not in the other two (GSE4573 and GSE31210; P>0.05; Table II). Additionally, a high level of FLT1 expression was only significantly
Table I. Cox regression analysis for high VEGFA mRNA expression in lung cancer cohorts.

\begin{tabular}{lcccr}
\hline Dataset & $\mathrm{n}$ & HR & $95 \%$ CI & P-value \\
\hline GSE3141 & 111 & 1.68 & $1.18-2.39$ & 0.004 \\
GSE4573 & 130 & 1.73 & $1.06-2.82$ & 0.028 \\
GSE8894 & 138 & 1.43 & $1.11-1.84$ & 0.006 \\
GSE31210 & 204 & 2.02 & $1.41-2.91$ & $<0.001$ \\
Combined & 583 & 1.94 & $1.50-2.50$ & $<0.001$
\end{tabular}

VEGFA, vascular epidermal growth factor A; HR, hazard ratio; CI, confidence interval.

Table II. Cox regression analysis for high FLT1 mRNA expression in lung cancer cohorts.

\begin{tabular}{lcccc}
\hline Dataset & $\mathrm{n}$ & HR & $95 \%$ CI & P-value \\
\hline GSE3141 & 111 & 1.66 & $1.01-2.73$ & 0.046 \\
GSE4573 & 130 & 1.11 & $0.63-1.95$ & 0.729 \\
GSE8894 & 138 & 1.69 & $1.02-2.08$ & 0.041 \\
GSE31210 & 204 & 1.30 & $0.84-2.01$ & 0.234 \\
Combined & 583 & 1.50 & $1.17-1.93$ & 0.002
\end{tabular}

FLT1, fms-related tyrosine kinase 1; HR, hazard ratio; CI, confidence interval.

Table III. Cox regression analysis for high KDR mRNA expression in lung cancer cohorts.

\begin{tabular}{lcccc}
\hline Dataset & $\mathrm{n}$ & HR & $95 \%$ CI & P-value \\
\hline GSE3141 & 111 & 1.01 & $0.76-1.30$ & 0.972 \\
GSE4573 & 130 & 0.96 & $0.66-1.39$ & 0.812 \\
GSE8894 & 138 & 1.23 & $1.01-1.49$ & 0.040 \\
GSE31210 & 204 & 0.92 & $0.68-1.25$ & 0.598 \\
Combined & 583 & 1.22 & $0.95-1.56$ & 0.127 \\
\hline
\end{tabular}

KDR, kinase insert domain receptor; HR, hazard ratio; CI, confidence interval.

associated with a shorter survival time in the GSE8894 dataset $(\mathrm{P}=0.03$; Fig. 2C), but not in the other three independent datasets (Fig. 2A, B and D). When the four datasets were combined, a high level of FLT1 mRNA expression was identified to be significantly associated with a shorter patient survival time according to Kaplan-Meier (log-rank test, $\mathrm{P}=0.001$; Fig. 2E) and Cox regression (HR, 1.50; 95\% CI, 1.17-1.93; $\mathrm{P}=0.002$; Table II) analyses.

With respect to KDR mRNA expression (Table III; Fig. 3), a significant association with survival was only detected in the GSE8894 dataset, but not in the other three datasets nor in the combined cohort. Taken together, the current data indicate that VEGFA mRNA expression is a consistent prognostic marker 

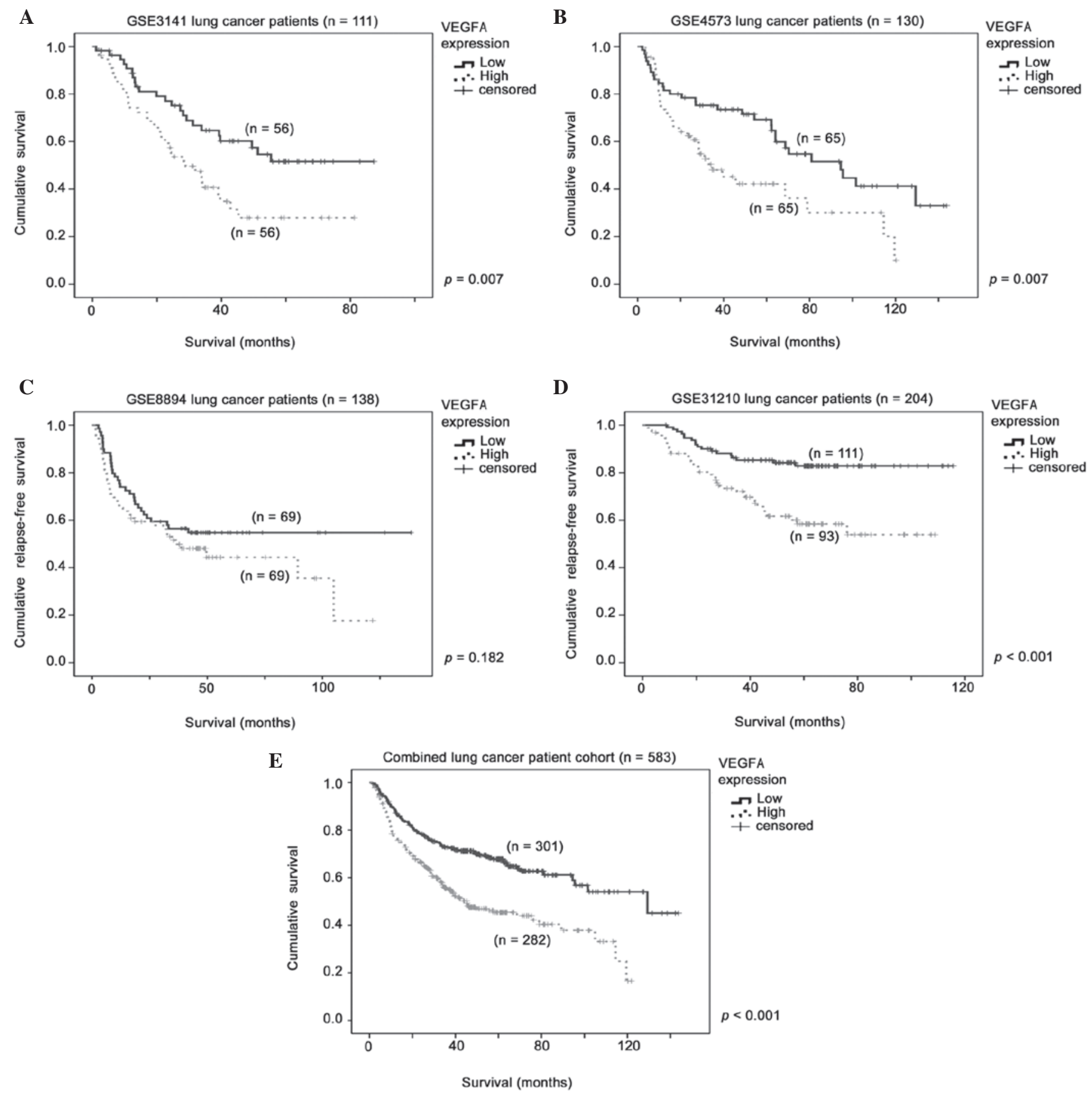

Figure 1. Association between VEGFA mRNA expression and the survival of patients with lung cancer. Kaplan-Meier analyses were performed for VEGFA mRNA expression in (A) GSE3141, (B) GSE4573, (C) GSE8894 and (D) GSE31210 datasets, and (E) the combined cohort. VEGFA, vascular epidermal growth factor A.

in patients with lung cancer, while FLT1 mRNA expression is a marginal prognostic marker and KDR mRNA expression is not a prognostic marker.

Positive correlation between VEGFA, FLT1 and VDR expression and lung cancer specimens. As VEGFA must bind to its receptors to generate downstream signaling, the overexpression of VEGFA alone may not be sufficient to drive tumor progression (19). Therefore, the present study investigated whether the expression of the ligand and its receptors are correlated. As indicated in Table IV, VEGFA
mRNA expression was significantly positively correlated with FLT1 mRNA expression in lung cancer specimens from all four independent cohorts investigated in the current study. Significant correlations between the expression of VEGFA and KDR, and FLT1 and KDR, were also observed in three out of four independent datasets (Table IV). The current results indicate that VEGFA and its receptors, FLT1 and KDR, may be co-regulated in tumors or in the tumor microenvironment.

High level of VEGFA and FLT1 mRNA expression in the same patients predicts a shorter survival outcome. As VEGFA and 
A GSE3141 lung cancer patients $(n=111)$

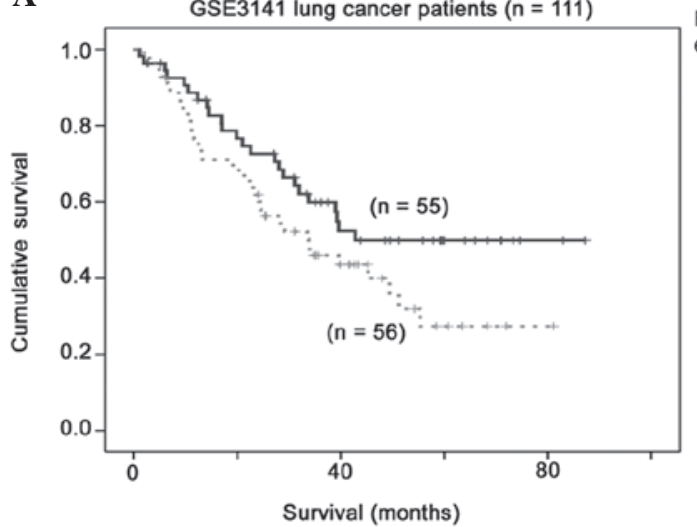

FLT expression

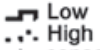

$\because$ High

$p=0.084$

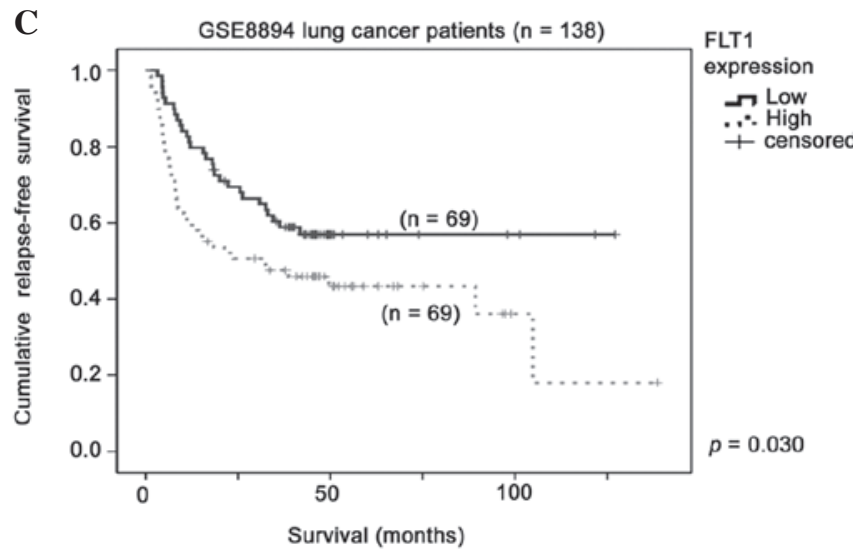

B

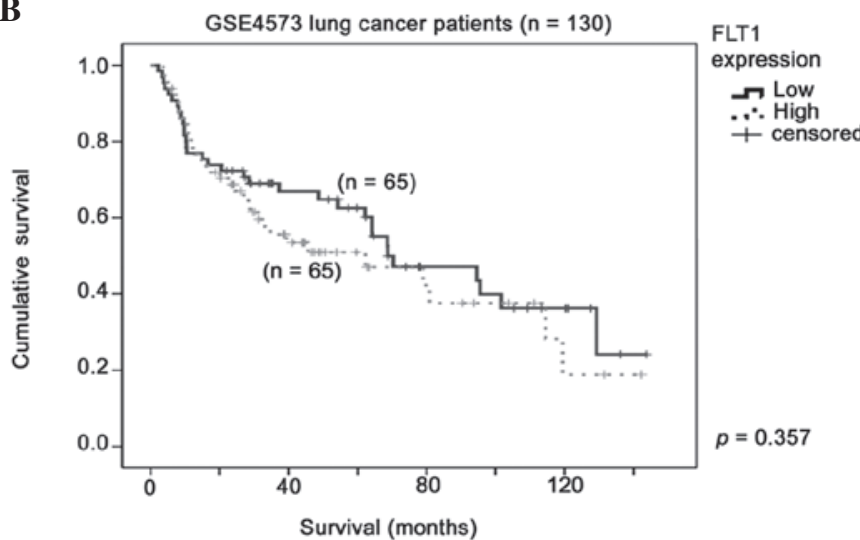

D

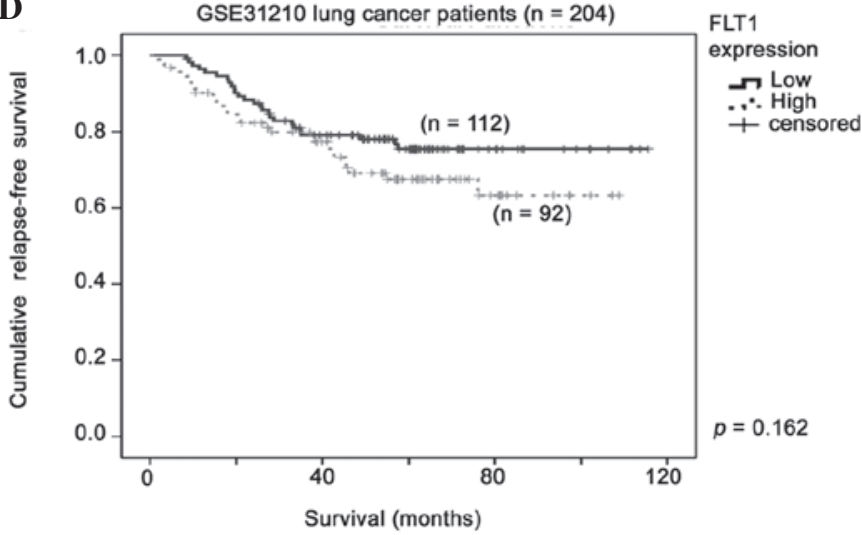

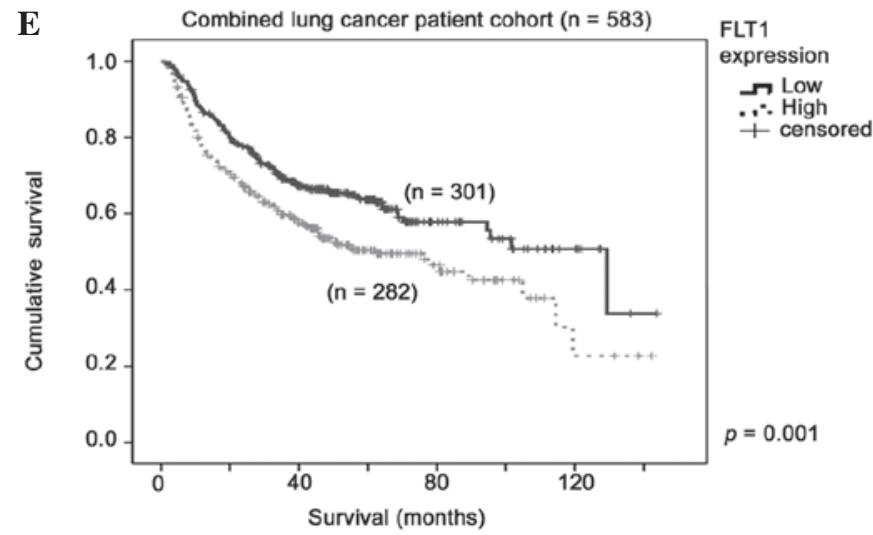

Figure 2. Association between FLT1 mRNA expression and the survival of patients with lung cancer. Kaplan-Meier analyses were performed for FLT1 mRNA expression in (A) GSE3141, (B) GSE4573, (C) GSE8894 and (D) GSE31210 datasets, and (E) the combined cohort. FLT1, fms-related tyrosine kinase 1.

FLT1 mRNA expression were significantly correlated in the four independent lung cancer patient cohorts (Table IV), the prognostic significance of the co-upregulation of these two factors was further investigated. Patients were stratified based on whether their expression of VEGFA and FLT1 mRNAs was high (above median) for both, high for one and low for the other (intermediate), or low for both. As indicated in Fig. 4, a high level expression of VEGFA and FLT1a expression was significantly associated with a shorter survival time in the GSE3141 ( $\mathrm{P}=0.007$; Fig. 4A), GSE4573 ( $\mathrm{P}=0.009$; Fig. 4B), GSE8894 ( $\mathrm{P}=0.031$; Fig. 4C) and GSE31210 ( $\mathrm{P}=0.003$; Fig. 4D) datasets. In the combined cohort, a high level of VEGFA mRNA expression was associated with a poor patient prognosis compared with high expression of a single factor $(\mathrm{P}<0.001$; Fig. 4E) or low expression of both factors ( $\mathrm{P}=0.045$; Fig. 4E).
High level of VEGFA and KDR mRNA expression in the same patients predicts a shorter survival outcome. Comparable results were obtained when VEGFA and KDR were similarly interrogated. A high level expression of the two factors was significantly associated with a poor prognosis in all four independent lung cancer patient cohorts $(\mathrm{P}<0.05$; Fig. 5A-D). Notably, in the combined cohort with a larger sample size, the patients with a high level of expression of the two factors were associated with a significantly shorter survival time compared with those exhibiting a low level of expression of either one of the two factors $(\mathrm{P}<0.001$; Fig. $5 \mathrm{E})$ or a low level of expression of both factors $(\mathrm{P}=0.002)$.

High level of VEGFA, FLT1 and KDR mRNA expression in the same patients predicts a shorter survival outcome. The 
Table IV. Correlation between the mRNA expression levels of VEGFA, FLT1 and KDR.

\begin{tabular}{lllll}
\hline Dataset & $\mathrm{n}$ & VEGFA vs. KDR & VEGFA vs. FLT1 & KDR vs. FLT1 \\
\hline GSE3141 & 111 & $\mathrm{r}=0.063, \mathrm{P}=0.512$ & $\mathrm{r}=0.218, \mathrm{P}=0.022^{\mathrm{a}}$ & $\mathrm{r}=0.678, \mathrm{P}<0.001^{\mathrm{a}}$ \\
GSE4573 & 130 & $\mathrm{r}=0.242, \mathrm{P}=0.005^{\mathrm{a}}$ & $\mathrm{r}=0.372, \mathrm{P}<0.001^{\mathrm{a}}$ & $\mathrm{r}=0.318, \mathrm{P}<0.001^{\mathrm{a}}$ \\
GSE8894 & 138 & $\mathrm{r}=0.371, \mathrm{P}<0.001^{\mathrm{a}}$ & $\mathrm{r}=0.361, \mathrm{P}<0.001^{\mathrm{a}}$ & $\mathrm{r}=0.143, \mathrm{P}=0.094$ \\
GSE31210 & 204 & $\mathrm{r}=0.394, \mathrm{P}<0.001^{\mathrm{a}}$ & $\mathrm{r}=0.684, \mathrm{P}<0.001^{\mathrm{a}}$ & $\mathrm{r}=0.469, \mathrm{P}<0.001^{\mathrm{a}}$ \\
\hline
\end{tabular}

${ }^{a} \mathrm{P}<0.05$. VEGFA, vascular epidermal growth factor A; FLT1, fms-related tyrosine kinase 1; KDR, kinase insert domain receptor.

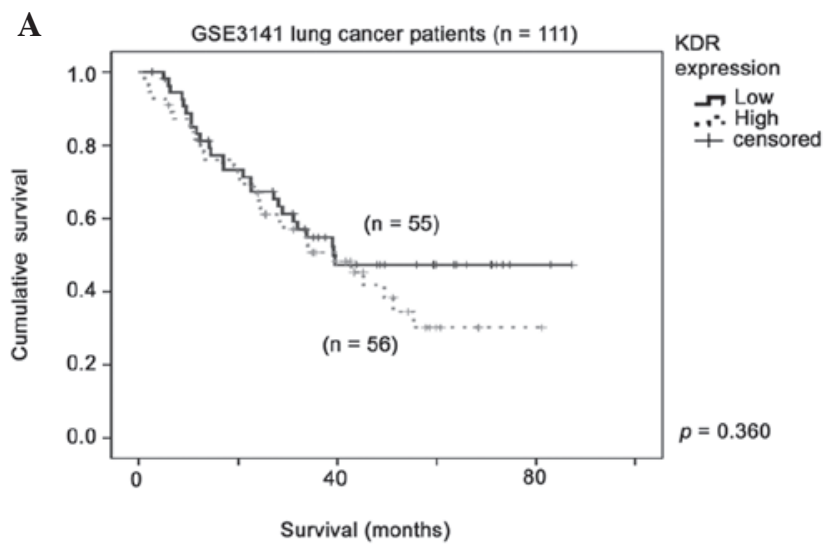

$\mathbf{B}$
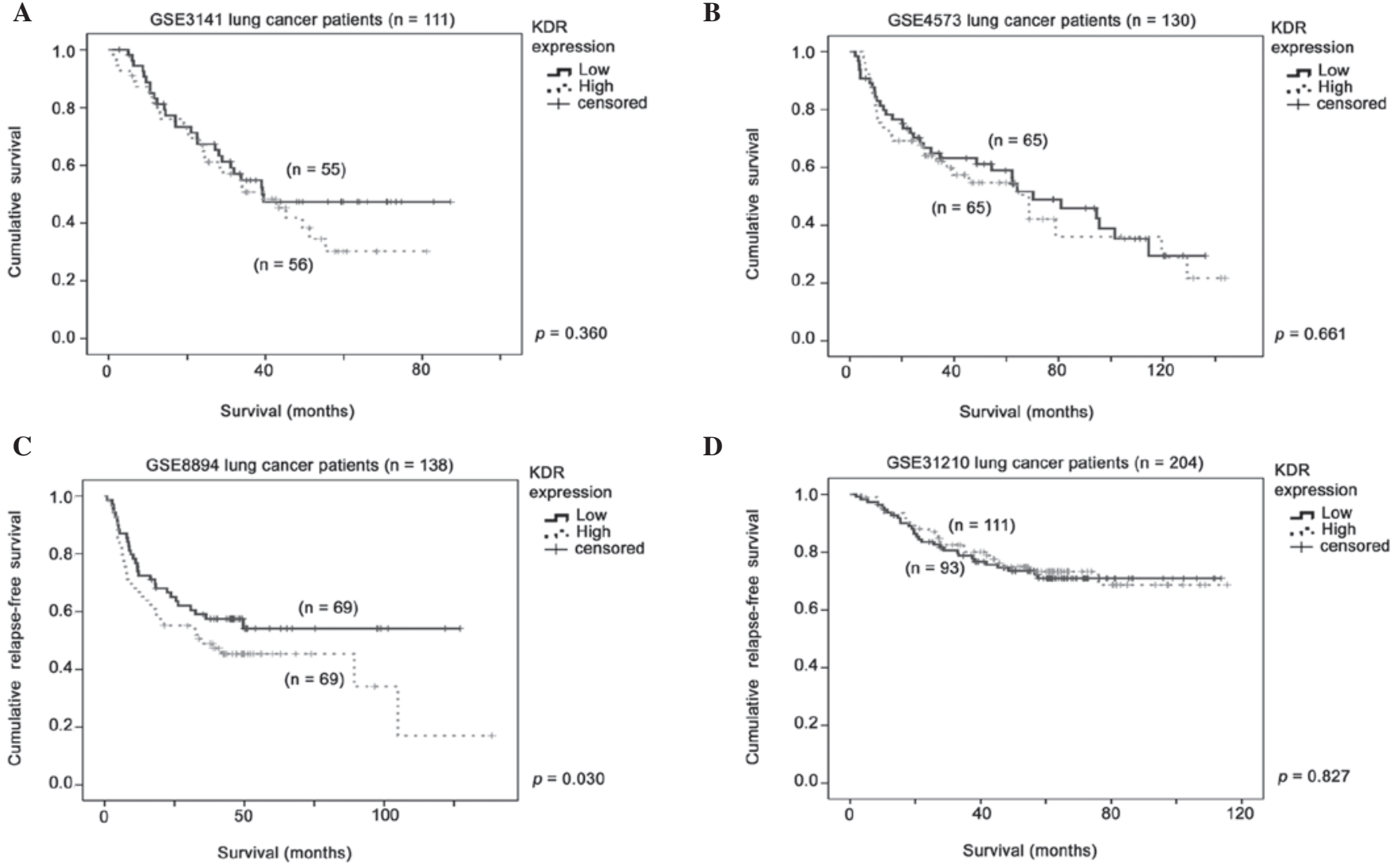

D
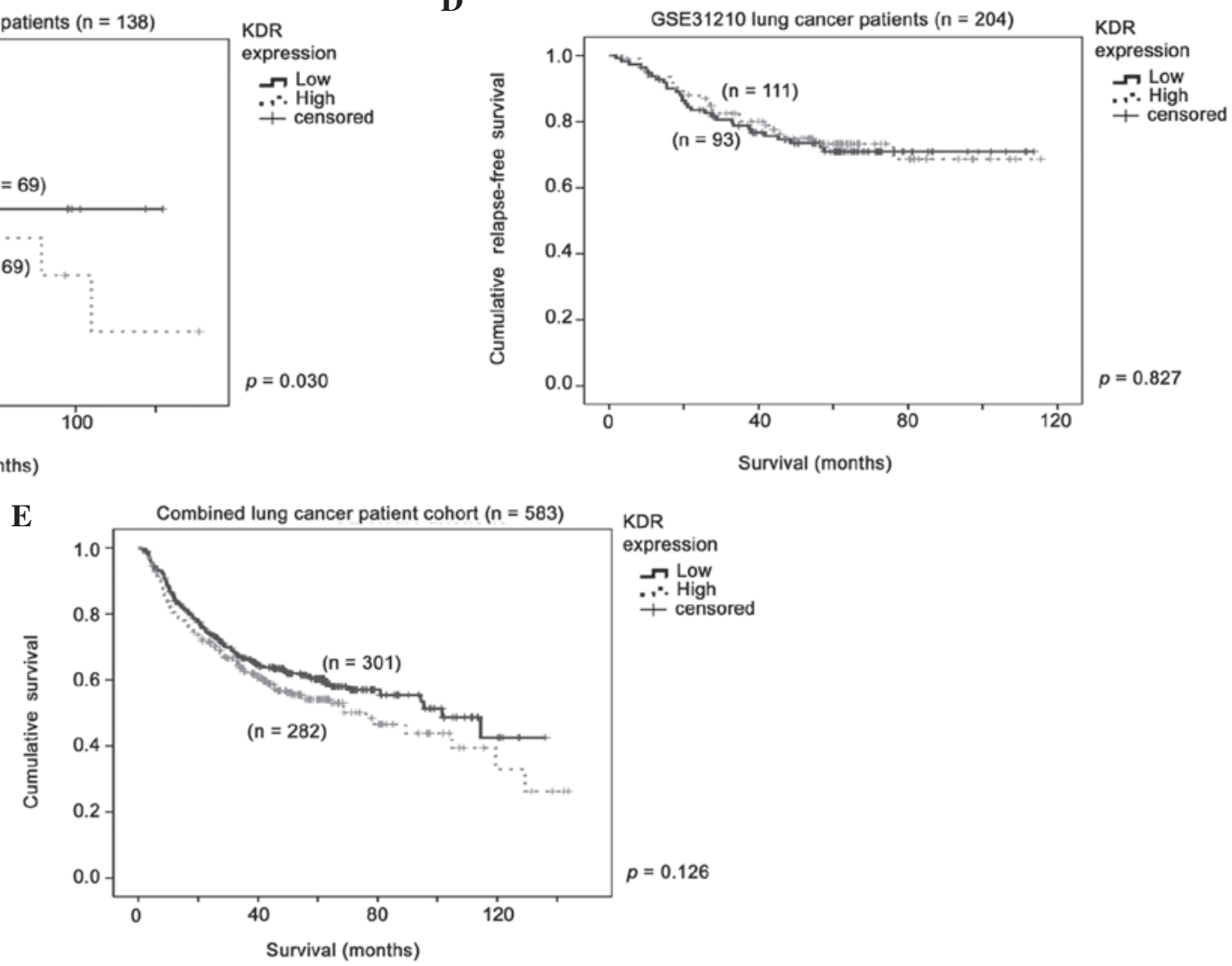

Figure 3. Association between KDR mRNA expression and the survival of patients with lung cancer. Kaplan-Meier analyses were performed for KDR mRNA expression in (A) GSE3141, (B) GSE4573, (C) GSE8894 and (D) GSE31210 datasets, and (E) the combined cohort. KDR, kinase insert domain receptor.

present study investigated whether VEGFA, FLT1 and KDR, which are involved in the same angiogenesis promoting signaling pathway, could be combined as a more effective prognostic indicator for patients with lung cancer. Notably, in the four independent cohorts, patients whose tumor specimens expressed a high level of all three factors exhibited a 

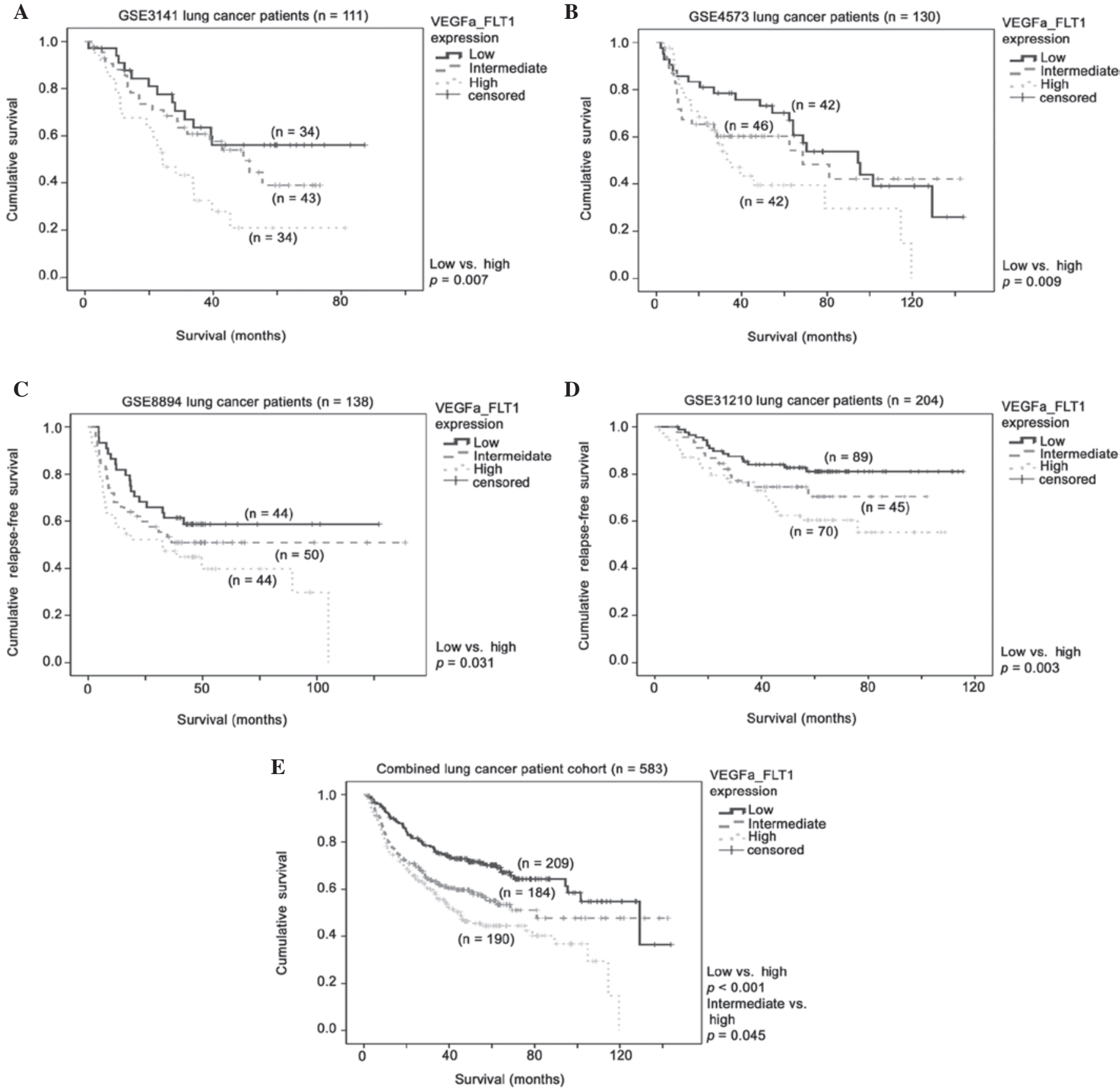

Figure 4. Association between combined VEGFA/FLT1 mRNA expression and the survival of patients with lung cancer. Kaplan-Meier analyses were performed for VEGFA and FLT1 mRNA expression in combination in (A) GSE3141, (B) GSE4573, (C) GSE8894 and (D) GSE31210 datasets, and (E) the combined cohort. VEGFA, vascular epidermal growth factor A; FLT1, fms-related tyrosine kinase 1.

significantly shorter survival compared with those expressing a low level of all these three factors $(\mathrm{P}<0.05$; Fig. 6A-D). In the combined cohort of 583 patients, the results were more pronounced. As indicated in Fig. 6E, the patients whose tumors overexpressed VEGFA, FLT1 and KDR exhibited significantly shorter survival times compared with those whose tumors overexpressed two $(\mathrm{P}=0.051)$, one $(\mathrm{P}=0.006)$ and none $(\mathrm{P}<0.001)$ of these factors. Furthermore, in the patients with lung cancer overexpressing all three, two or one of these factors, Cox regression analysis was used to determine the HRs of 2.27, 1.65 and 1.40, respectively, for a shorter relapse-free or overall survival time (Table V).

\section{Discussion}

A previous study attempted to combine angiogenic factors together for improved prognostification in patients with non-small cell lung cancer. In the study, a high level of VEGFA mRNA expression, and a low level of VEGFB and VEGFD mRNA expression was found to be associated with the poorest outcome (20). However, the functional roles and the biological importance of VEGFB and VEGFD suppression are largely unknown. By contrast, the co-upregulation of VEGFA, FLT1 and KDR mRNA expression in the tumor environment identified in the present study is supported by the well-known 

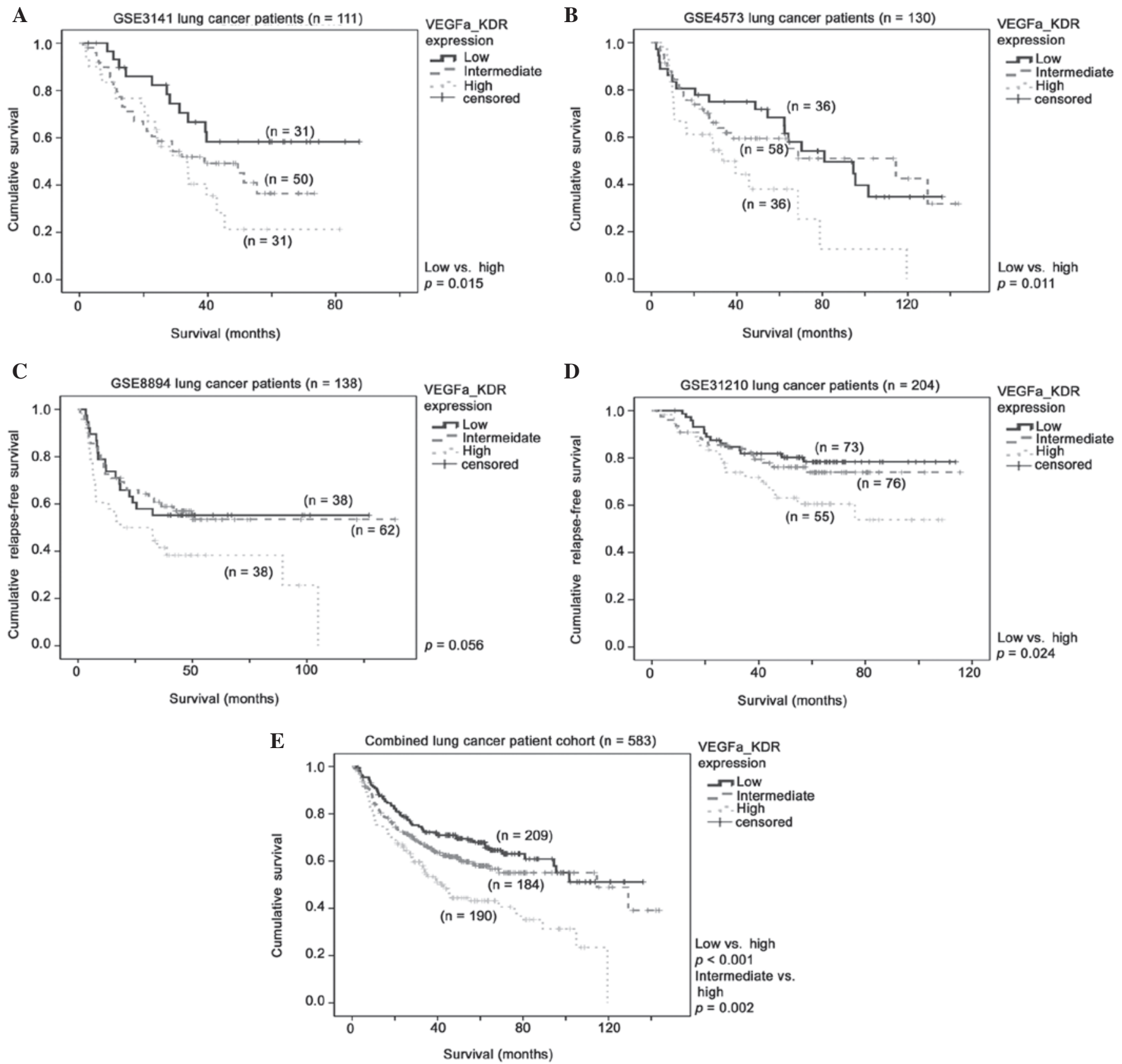

Figure 5. Association between combined VEGFA/KDR mRNA expression and the survival of patients with lung cancer. Kaplan-Meier analyses were performed for VEGFA and KDR mRNA expression in combination in (A) GSE3141, (B) GSE4573, (C) GSE8894 and (D) GSE31210 datasets, and (E) the combined cohort. VEGFA, vascular epidermal growth factor A; KDR, kinase insert domain receptor.

Table V. Cox regression analysis for VEGFA/FLT1/KDR mRNA overexpression in the combined cohort $(\mathrm{n}=583)$.

\section{Overexpressed}

genes, $\mathrm{n}$

HR

$95 \% \mathrm{CI}$

P-value

\section{0}

1

2

3

Reference
1.40
1.65
2.27

0.96-2.05

0.083

$1.15-2.37$

0.007

$1.57-3.28$

$<0.001$

VEGFA, vascular epidermal growth factor A; FLT1, fms-related tyrosine kinase 1; KDR, kinase insert domain receptor; HR, hazard ratio; $\mathrm{CI}$, confidence interval. interaction between VEGFA and the two receptors, FLT1 and $\mathrm{KDR}$, though which downstream signaling for angiogenesis is activated $(5,21)$.

Previous studies have produced contradicting results regarding the prognostic significance of VEGFA, FLT1 and KDR expression in lung cancer $(12,13)$. In the present analysis, it was identified that only VEGFA mRNA expression, but not FLT1 and KDR expression, was consistently significantly associated with patient survival. Notably, and in contrast to previous studies $(12,13)$, a high level of FLT1 mRNA expression was significantly associated with poorer survival in the combined cohort of 583 patients. By combining VEGFA and FLT1 mRNA expression, it was identified that tumors overexpressing these two factors had a poorer prognosis compared with tumors 

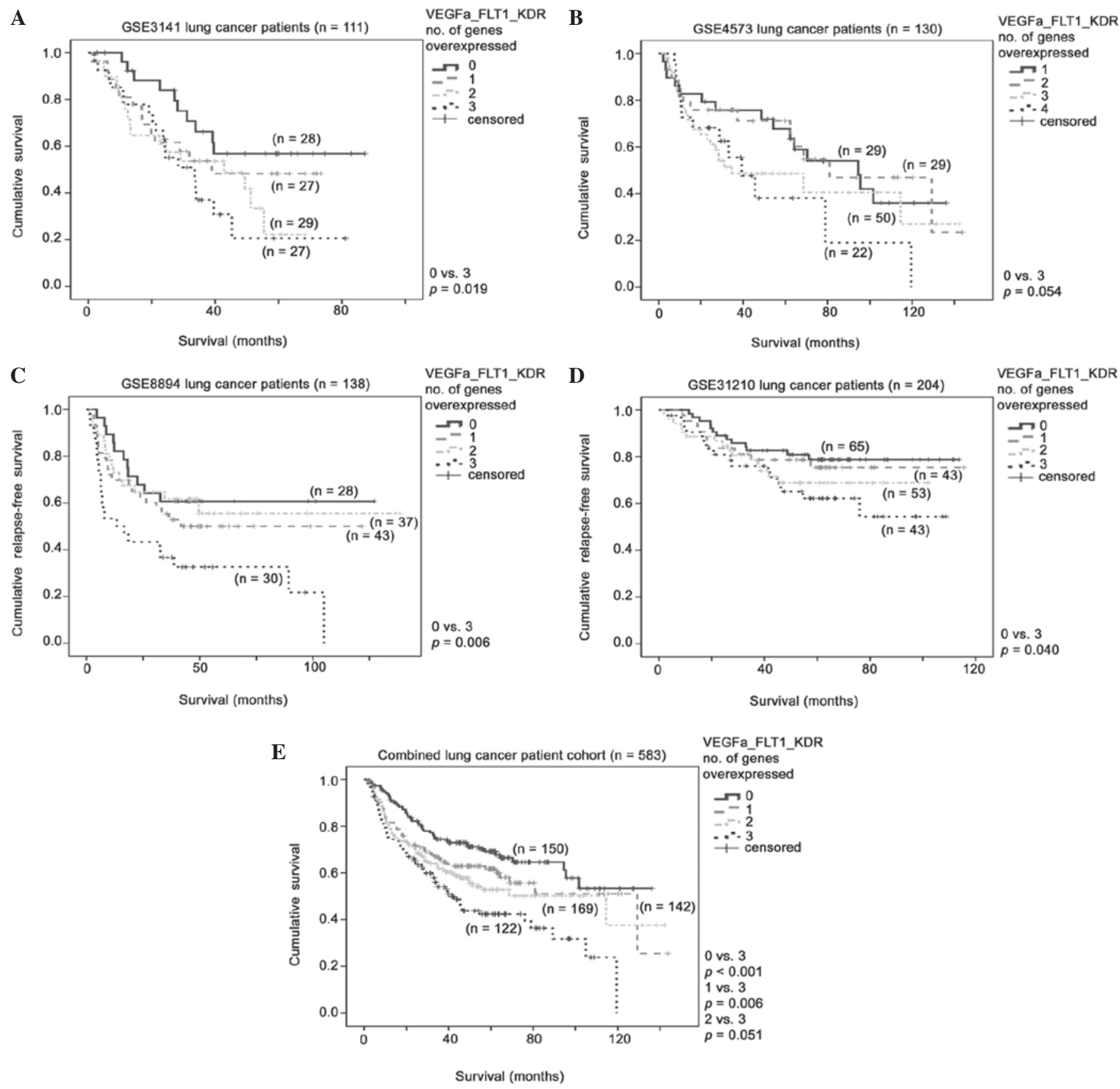

Figure 6. Association between combined VEGFA/FLT1/KDR mRNA expression and the survival of patients with lung cancer. Kaplan-Meier analyses were performed for VEGFA, FLT1 and KDR mRNA expression in combinaton in (A) GSE3141, (B) GSE4573, (C) GSE8894 and (D) GSE31210 datasets, and (E) the combined cohort. VEGFA, vascular epidermal growth factor A; FLT1, fms-related tyrosine kinase 1; KDR, kinase insert domain receptor.

overexpressing only one or neither of these two factors. By contrast, KDR mRNA expression itself was not significantly associated with survival in the 583 patients with lung cancer. However, combined VEGFA, FLT1 and KDR mRNA expression was a consistent and statistically significant prognostic indicator for lung cancer in the four independent lung cancer patient cohorts examined, as well as in the combined cohort. Therefore, the current results prime future investigations into whether this combination could predict the sensitivity of the patients to agents that target angiogenesis, including bevacizumab or nintedanib, which have demonstrated promising results in phase III clinical trials in patients with lung cancer $(22,23)$.

Various studies have demonstrated that the expression of VEGFA in fibroblasts and immune cells surrounding the tumor mass may also be important in cancer progression, in addition to VEGFA in the tumor cells themselves $(24,25)$. The results from these studies indicate that it may not be sufficient to analyze only the tumor expression of VEGFA, FLT1 and KDR. Rather, their expression levels at the tumor site as a whole may be more important, as this widespread expression accounts for the autocrine and paracrine actions of the VEGF signaling pathway, which drive angiogenesis, and tumor growth and survival. Similar results have been observed in colon cancer, suggesting that co-consideration of these factors may have prognostic implication for multiple cancer types that required angiogenesis for tumor progression (26).

Although the cohorts included in the present study were highly heterogeneous in terms of patient characteristics, 
specimen procurement, RNA extraction, treatment and data collection, the observations of VEGFA, and combined VEGFA, FLT1 and KDR mRNA expression, were highly consistent between these four independent cohorts, indicating that this combination may be highly relevant and readily applicable in clinical practice as a more effective prognostic indicator for patients with lung cancer. However, additional investigations are required to demonstrate the clinical applicability of combined VEGFA/FLT1/KDR expression, as well as its predictive value as an anti-angiogenic agent, for example, by conducting a prospective trial with or without application of an agent targeting angiogenesis.

\section{Acknowledgements}

The authors would like to thank Omic Science and Technology Ltd. (Hong Kong, SAR, China) for performing the initial gene analysis and for their support in editing of the original manuscript. The present study was supported by the University of Macau Start-Up Research Grant (no. SRG2014-00006-FHS) and Multi-Year Research Grant (no. MYRG2015-00065-FHS).

\section{References}

1. Hicklin DJ and Ellis LM: Role of the vascular endothelial growth factor pathway in tumor growth and angiogenesis. J Clin Oncol 23: 1011-1027, 2005

2. Knizetova P, Ehrmann J, Hlobilkova A, Vancova I, Kalita O, Kolar Z and Bartek J: Autocrine regulation of glioblastoma cell cycle progression, viability and radioresistance through the VEGF-VEGFR2 (KDR) interplay. Cell Cycle 7: 2553-2561, 2008.

3. Lichtenberger BM, Tan PK, Niederleithner H, Ferrara N, Petzelbauer P and Sibilia M: Autocrine VEGF signaling synergizes with EGFR in tumor cells to promote epithelial cancer development. Cell 140: 268-279, 2010.

4. Hamerlik P, Lathia JD, Rasmussen R, Wu Q, Bartkova J, Lee M, Moudry P, Bartek J Jr, Fischer W, Lukas J, et al: Autocrine VEGF -VEGFR2-Neuropilin-1 signaling promotes glioma stem-like cell viability and tumor growth. J Exp Med 209: 507-520, 2012.

5. Bieche I, Vacher S, Vallerand D, Richon S, Hatem R, De Plater L, Dahmani A, Némati F, Angevin E, Marangoni E, et al: Vasculature analysis of patient derived tumor xenografts using species-specific PCR assays: Evidence of tumor endothelial cells and atypical VEGFA-VEGFR1/2 signalings. BMC Cancer 14: 178, 2014

6. Andersen S, Donnem T, Al-Shibli K, Al-Saad S, Stenvold H, Busund LT and Bremnes RM: Prognostic impacts of angiopoietins in NSCLC tumor cells and stroma: VEGF-A impact is strongly associated with Ang-2. PLoS One 6: e19773, 2011.

7. Jantus-Lewintre E, Sanmartín E, Sirera R, Blasco A, Sanchez JJ, Tarón M, Rosell $\mathrm{R}$ and Camps C: Combined VEGF-A and VEGFR-2 concentrations in plasma: Diagnostic and prognostic implications in patients with advanced NSCLC. Lung Cancer 74: 326-331, 2011.

8. Donnem T, Al-Shibli K, Andersen S, Al-Saad S, Busund LT and Bremnes RM: Combination of low vascular endothelial growth factor A (VEGF-A)/VEGF receptor 2 expression and high lymphocyte infiltration is a strong and independent favorable prognostic factor in patients with nonsmall cell lung cancer. Cancer 116: 4318-4325, 2010.

9. Takenaka K, Katakura H, Chen F, Ogawa E, Adachi M, Wada H and Tanaka F: The ratio of membrane-bound form Flt-1 mRNA to VEGF mRNA correlates with tumor angiogenesis and prognosis in non-small cell lung cancer. Cancer Lett 246: 34-40, 2007.
10. Roybal JD, Zang Y, Ahn YH, Yang Y, Gibbons DL, Baird BN, Alvarez C, Thilaganathan N, Liu DD, Saintigny P, et al: miR-200 Inhibits lung adenocarcinoma cell invasion and metastasis by targeting Flt1/VEGFR1. Mol Cancer Res 9: 25-35, 2011.

11. An SJ, Nie Q, Chen ZH, Lin QX, Wang Z, Xie Z, Chen SL, Huang Y, Zhang AY, Yan JF, et al: KDR expression is associated with the stage and cigarette smoking of the patients with lung cancer. J Cancer Res Clin Oncol 133: 635-642, 2007.

12. Seto T, Higashiyama M, Funai H, Imamura F, Uematsu K, Seki N, Eguchi K, Yamanaka T and Ichinose Y: Prognostic value of expression of vascular endothelial growth factor and its flt- 1 and KDR receptors in stage I non-small-cell lung cancer. Lung Cancer 53: 91-96, 2006.

13. Volm M, Koomägi R and Mattern J: Prognostic value of vascular endothelial growth factor and its receptor Flt-1 in squamous cell lung cancer. Int J Cancer 74: 64-68, 1997.

14. Bild AH, Yao G, Chang JT, Wang Q, Potti A, Chasse D, Joshi MB, Harpole D, Lancaster JM, Berchuck A, et al: Oncogenic pathway signatures in human cancers as a guide to targeted therapies. Nature 439: 353-357, 2006.

15. Raponi M, Zhang Y, Yu J, Chen G, Lee G, Taylor JM, Macdonald J, Thomas D, Moskaluk C, Wang Y, et al: Gene expression signatures for predicting prognosis of squamous cell and adenocarcinomas of the lung. Cancer Res 66: 7466-7472, 2006.

16. Lee ES, Son DS, Kim SH, Lee J, Jo J, Han J, Kim H, Lee HJ Choi HY, Jung Y, et al: Prediction of recurrence-free survival in postoperative non-small cell lung cancer patients by using an integrated model of clinical information and gene expression. Clin Cancer Res 14: 7397-7404, 2008.

17. Okayama H, Kohno T, Ishii Y, Shimada Y, Shiraishi K, Iwakawa R, Furuta K, Tsuta K, Shibata T, Yamamoto S, et al: Identification of genes upregulated in ALK-positive and EGFR/KRAS/ALK-negative lung adenocarcinomas. Cancer Res 72: 100-111, 2012.

18. Yuen HF, Gunasekharan VK, Chan KK, Zhang SD, Platt-Higgins A, Gately K, O'Byrne K, Fennell DA, Johnston PG, Rudland PS, et al: RanGTPase: A candidate for Myc-mediated cancer progression. J Natl Cancer Inst 105: 475-488, 2013.

19. Ferrara N: VEGF and the quest for tumour angiogenesis factors. Nat Rev Cancer 2: 795-803, 2002.

20. Sanmartín E, Sirera R, Usó M, Blasco A, Gallach S, Figueroa S, Martínez N, Hernando C, Honguero A, Martorell M, et al: A gene signature combining the tissue expression of three angiogenic factors is a prognostic marker in early-stage non-small cell lung cancer. Ann Surg Oncol 21: 612-620, 2014.

21. Vieira JM, Ruhrberg C and Schwarz Q: VEGF receptor signaling in vertebrate development. Organogenesis 6: 97-106, 2010.

22. Sandler A, Gray R, Perry MC, Brahmer J, Schiller JH, Dowlati A, Lilenbaum R and Johnson DH: Paclitaxel-carboplatin alone or with bevacizumab for non-small-cell lung cancer. N Engl J Med 55: 2542-2550, 2006.

23. Reck M, Kaiser R, Mellemgaard A, Douillard JY, Orlov S, Krzakowski M, von Pawel J, Gottfried M, Bondarenko I, Liao M, et al; LUME-Lung 1 Study Group: Docetaxel plus nintedanib versus docetaxel plus placebo in patients with previously treated non-small-cell lung cancer (LUME-Lung 1): A phase 3 , double-blind, randomised controlled trial. Lancet Oncol 15: 143-155, 2014.

24. Fukumura D, Xavier R, Sugiura T, Chen Y, Park EC, Lu N, Selig M, Nielsen G, Taksir T, Jain RK, et al: Tumor induction of VEGF promoter activity in stromal cells. Cell 94: 715-725, 1998.

25. Liang WC, Wu X, Peale FV, Lee CV, Meng YG, Gutierrez J, Fu L, Malik AK, Gerber HP, Ferrara N, et al: Cross-species vascular endothelial growth factor (VEGF)-blocking antibodies completely inhibit the growth of human tumor xenografts and measure the contribution of stromal VEGF. J Biol Chem 281: 951-961, 2006.

26. Zhang SD, McCrudden CM, Meng C, Lin Y and Kwok HF: The signifiance of combining VEGFA, FLT1 and KDR expressions in colon cancer patient prognosis and predicting response to bevacizumab. Onco Targets Ther 8: 835-843, 2015. 